\title{
Error Behaviour in PID Controll Systems with Dynamic Processes
}

\author{
František Kudlačák \\ Faculty of Informatics and Information \\ Technologies, \\ Slovak University of Technology, \\ Bratislava, Slovakia \\ frantisek.kudlacak@stuba.sk
}

\author{
Tibor Krajčovič \\ Faculty of Informatics and Information \\ Technologies, \\ Slovak University of Technology, \\ Bratislava, Slovakia \\ tibor.krajcovic@stuba.sk
}

\begin{abstract}
This paper deals with current state of art in field of tuning methods for PID controllers, and shows how output error depends on dynamicity of the process. Controlled process is dynamic, therefore it can change over with various dynamicity. This dependency leads to adaptive controllers, and new tuning methods for them. In the first part of the paper is presented general description of PID controller and tuning algorithms. There are mentioned online and offline tuning methods. In the second part of the paper, is mentioned first experimental result, which shows dependency of output error on dynamicity of process. This dependency has serious effects on behaviour of whole control system.
\end{abstract}

Keywords- error dependancy; dynamic process; PID controll systems

\section{INTRODUCTION}

Control systems are well established in industry nowadays. They can control smallest robots or big factory or machines. Control systems and feedback elements were separated. In 1970s revolution in control systems was held. Feedback elements and control systems were integrated into one device, so complex problems could be solved within integrated system. This revolution was driven by the new breakthroughs in the field of embedded systems. Improvement in this field allows integration of control and feedback element. Open loop systems were transformed into closed loop systems. For example automobile emissions have decreased by 90 percent over last 25 years due to specialized embedded systems, which are developed to control engine. In modern car there can be found more than 100 separate embedded systems, which communicate together. Modern embedded system can include not only computing subsystems, but it can also include hardware and other mechanical parts or actuators.

Controlled processes are different. If the process is static and it does not change over the time, the controller does not have to change and if it is well designed, the process works well. But if the process has disturbances on output or process changes over time, and dynamicity of these changes change as well, the control system should adapt.

\section{PID CONTROLLER}

The most common control systems in industrial controllers utilize Proportional-Integral-Derivative
(PID) control logic [1]. PID controller calculates error of the system and in each loop, PID controller attempts to decrease error value of the system by adjusting control input to the process (see Fig. 1).

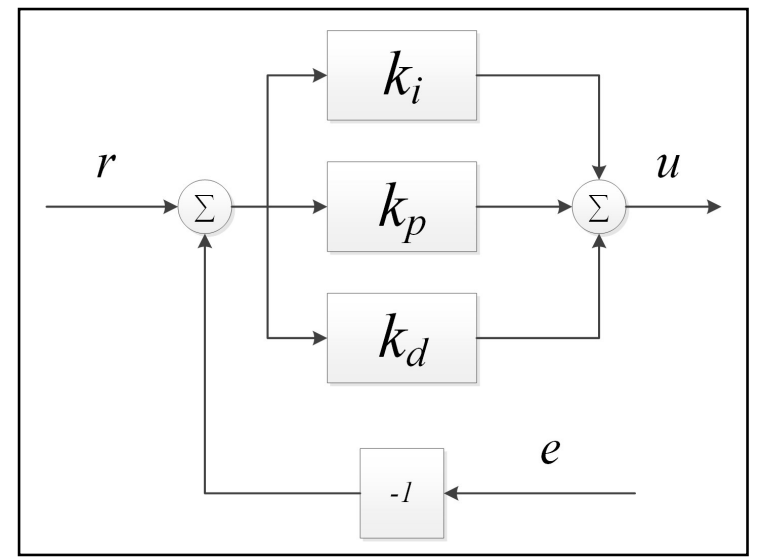

Figure 1. PID controller.

Where $r$ is reference signal called setpoint, $u$ is control signal for process which adjust input signal to process. Element $e$ is error element, and it is calculated as difference between process output and desired setpoint $r$. Each element of PID controller is weighted. Proportional part is weighted with parameter $k_{p}$, integral part is weighted with $k_{i}$, and derivative part is weighted with parameter $k_{d}$. These parameters are called gains. Desciption of PID controller can be represented by folowing formula (1):

$$
\begin{aligned}
& u=k_{p} e+k_{i} \int_{0}^{t} e(\tau) d \tau+k_{d} \frac{d e}{d t} \\
& u=k_{p}\left(e+\frac{1}{T_{i}} \int_{0}^{t} e(\tau) d \tau+T_{d} \frac{d e}{d t}\right)
\end{aligned}
$$

Where $u$ is control signal for the process, $e$ is error of the process $\tau$ is time constant $k_{i}, k_{p}, k_{d}$ are gains, $T_{i}$ is interval for integral part of PID controller and $T_{d}$ is derivative time for PID controller. Parameters $k_{i}, k_{p}, k_{d}$ can be tuned by tuning methods. There are many tuning methods, and they use different approaches. First type of tuning methods is based on professional 
estimation of PID parameters. Other tuning methods are based on time when tuning parameters are set. If they are set outside of the plant loop, they are called offline tuning methods, but if they are set inside the plant loop, they are called online tuning methods.

\section{OfFline TUNING MethodS}

Offline tuning methods are based on setting parameters before planting process into execution. Parameters are not changing during the execution of the process, they can be changed only if the execution of the process is interrupted and setting parameters does not reflect behaviour of the process automatically. If the set parameters are wrong or they are not sufficient, the process is interrupted and the parameters have to be set again. There are described some offline tuning methods.

\section{A. Professional Estimation of Parameters}

Parameters of PID controller are set by experienced person with proper knowledge of regulated process. If the set parameters are wrong and PID controller does not work properly, the parameters have to be adjusted adequately. This approach is slow in view of acquiring the best parameters for PID controller. This method cannot reflect dynamic process.

\section{B. Dominant Pole Assigment Tuning Method}

This method is applied to a test group of process behaviours. There need to be found correlation between behaviour of process and the parameters of PID controller. Dominant poles are assigned as integration of process error. The setpoint weighting is used to improve setpoint response of system [2].

\section{Dominant Pole Assigment Tuning Method with Use of Genetic Algorhitm}

Parameters of dominant pole assignment tuning methods can be improved by genetic algorithm [3]. Formula (1) can be rewritten into following form (2):

$$
u=K\left[\left(b y_{r}-y\right)+\frac{1}{T_{i}} \int e d t+T_{d} \frac{d e}{d t}\right]
$$

Where $u$ is controller output, $y_{r}$ is set-point, $y$ is process output, $K$ is controller gain, $b$ is set-point weighting, $T_{i}$ is integral time, $T_{d}$ is derivative time. Parameters $K, T_{i}, T_{d}, b$ are considered as one individual in a population. Initial population is chosen randomly. And afterwards fitness, for each individual in population, is evaluated. If final condition is matched, algorithm is stopped and there are used final parameters for PID controller, if not there are applied genetic operations on individuals and there is created new population. For applying genetic algorithm there need to be a model of process and proper test batch. This enhanced method can be executed automatically, and provides sufficient results for static processes with low disturbance.

\section{Ziegle-Nichols Tuning Method}

Is heuristic method [4]. Provides sufficient results if there is known delay function of process. If there is process with transportation delay (Fig. 2) there can be derive parameters $\mathrm{L}$ and $\mathrm{T}$ by drawing a tangent at the point of intersection with time axis and with stable state value.

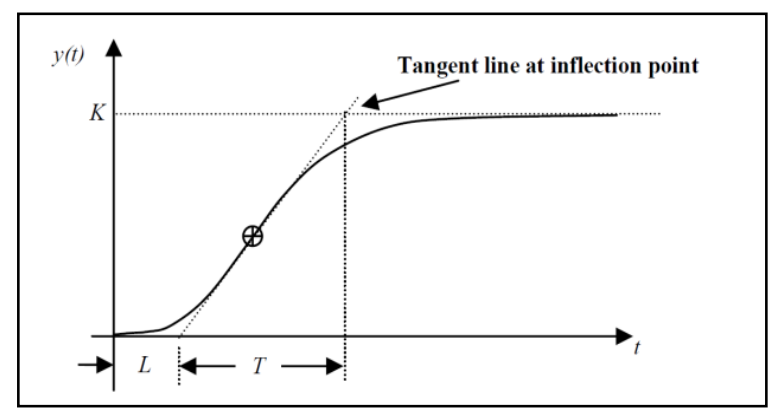

Figure 2. Response curve for Ziegler-Nichols method [5].

Parameters of PID controller are afterwards computed from Tab. 1:

TABLE I. PARAMETERS ACCORDING TO ZIEGLER-NICHOLS TUNING METHOD [5].

\begin{tabular}{|c|c|c|c|}
\hline Controller type & $\mathrm{Kp}$ & $\mathrm{Ti}$ & $\mathrm{Td}$ \\
\hline $\mathrm{P}$ & $\mathrm{T} / \mathrm{L}$ & & \\
\hline $\mathrm{PI}$ & $0.9 \mathrm{~T} / \mathrm{L}$ & $\mathrm{L} / 0.3$ & \\
\hline $\mathrm{PID}$ & $1.2 \mathrm{~T} / \mathrm{L}$ & $2 \mathrm{~L}$ & $0.5 \mathrm{~L}$ \\
\hline
\end{tabular}

\section{E. Particle Swarm Optimization Tuning Method}

PSO tuning method is based on numerical functions. This method uses as representation of results with particle in D-dimensional space, which is defined by position, velocity and the best result [6]. D is number of parameters of PID controller. Each particle is defined by three parameters (3):

$$
\begin{aligned}
& x_{i}=\left(x_{i 1}, x_{i 2}, \ldots, x_{i D}\right) \\
& v_{i}=\left(v_{i 1}, v_{i 2}, \ldots, v_{i D}\right) \\
& p_{i}=\left(p_{i 1}, p_{i 2}, \ldots, p_{i D}\right)
\end{aligned}
$$

Where $x_{i}$ represents position of particle in Ddimensional space, $v_{i}$ is speed of particle in Ddimensional space and $p_{i}$ is best result of particle in Ddimensional space. In first step there is generated swarm of particle which can be created randomly. Then fit function is computed for each particle. Fit function is computed from position of particle, and position represents parameters of PID controller. If finish criteria is matched particle swarm optimization algorithm ends, if criteria is not matched, there is computed next generation. Position of particle is computed from the last position and speed as vector addition. Speed of particle is based on the best solution, position and speed (4). 


$$
v_{i d}=w v_{i d}+c_{1} r\left(p_{i d}-x_{i d}\right)+c_{2} R\left(p_{g d}-x_{i d}\right)
$$

Where $w$ is weight of speed, and it can change over time, $p_{g d}$ is position of the nearest partition, $c_{1}$ and $c_{2}$ are positive constants, and $r$ and $R$ are random functions from 0 to 1 , both included [7].

\section{OnLine Tuning Methods}

If process is running and parameters are set during execution, these methods are called online tuning methods. They can react to changes in process and adjust PID parameters according to the changes. If process is dynamic or there is no description of process, offline tuning methods are not sufficient. There are described some online tuning methods.

\section{A. Two Layer Tuning Method}

Dynamicity of many plant processes can be high, due to characteristics of process itself or due to delays or disturbances. Ignoring these facts can lead to unexpected behaviour of controlled system. If behaviour of process is nonlinear, advanced techniques are required. Two layer method is based on dividing solution into two steps. In the first step boundaries for the parameters of PID controller are computed. In the second step are analytically selected values from computed boundaries. Boundaries are computed offline, with use of modified offline tuning methods. Schematic representation (see Fig. 3) of two level system includes classical tuning method block and optimization block, which utilizes model of nonlinear controller.

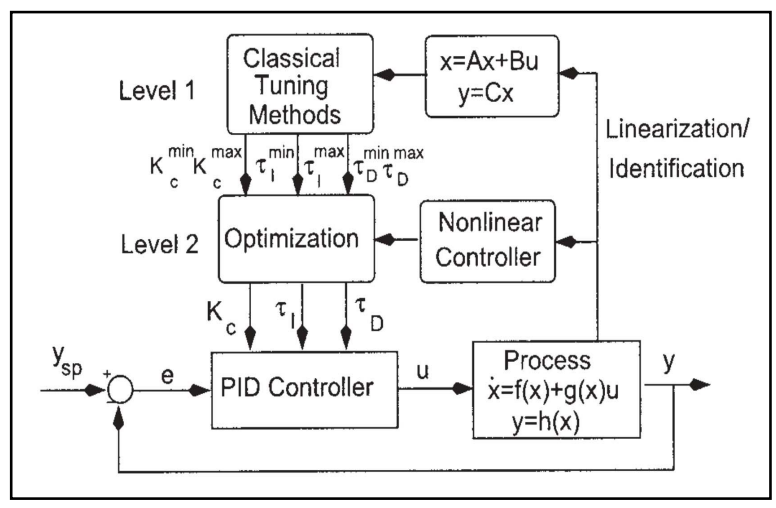

Figure 3. Two level optimization based tuning method [8].

\section{B. Internal Model Tuning Methods}

This methods require mathematical model of controlled process. Effectiveness of this method depends on the mathematical model. If model is precise, this tuning methods is robust and with very good results [9].

\section{EXPERIMENTAL RESULTS}

Error behavioral was tested on following set points (Fig. 4). For set point function there were chosen different types. Firstly was tested traditional set point in one value, it was followed by liner function of set points and with two different exponential functions. This data set represents much more set point cases, than only traditional set point use case.

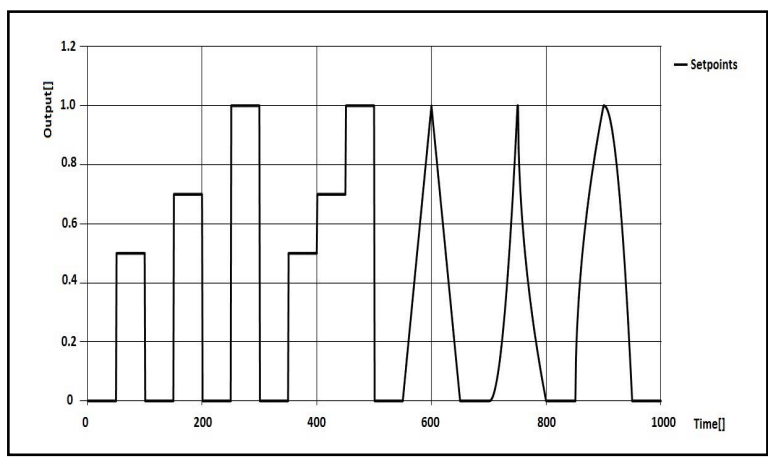

Figure 4. Set points.

For simulation we determine following processes. (Fig. 5, Fig. 6, Fig. 7).

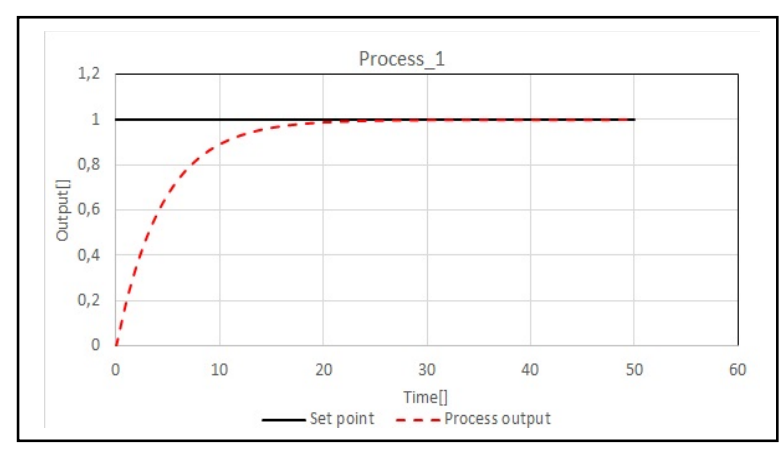

Figure 5. First process.

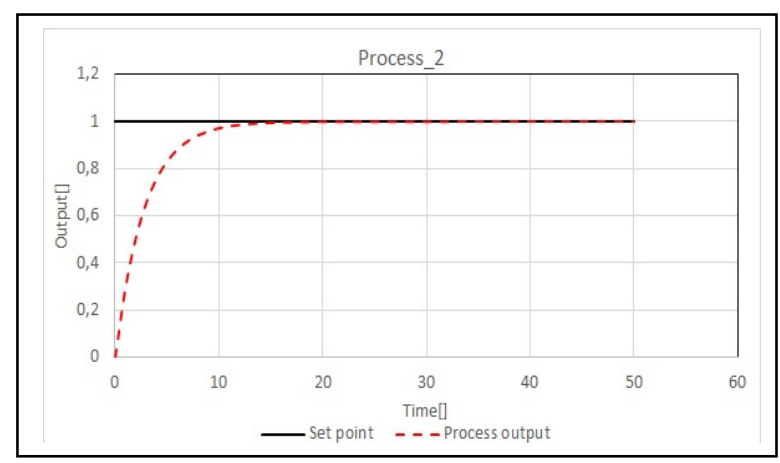

Figure 6. Second process.

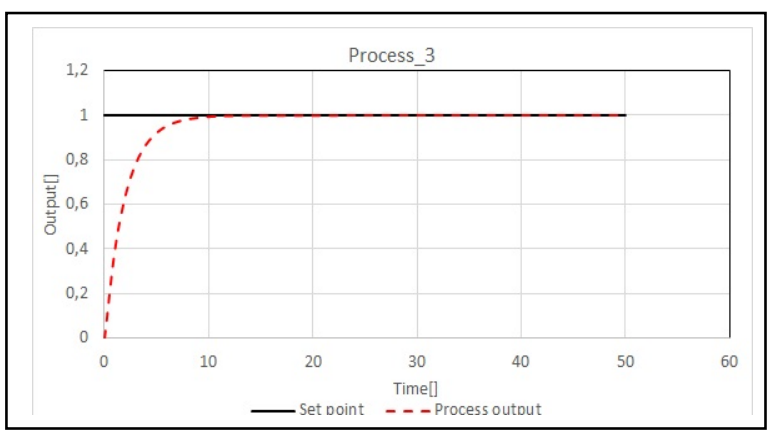

Figure 7. Third process. 
All three processes are similar in term of behavior but there is increased delay. First process is delayed by $10 \%$ according to second and $20 \%$ according to third.

After setting test processes, there were tuned parameters for PID controller. It was tuned for first process and there was used evolution algorithm to tune these parameters. $\mathrm{P}$ parameter was set to 4.0 , I parameter was set to 3.0 and D parameter was set to 0.0. This tuned PID controller was used in all three cases and its behavior is shown in following figures. (Fig. 8, Fig. 9, Fig. 10)

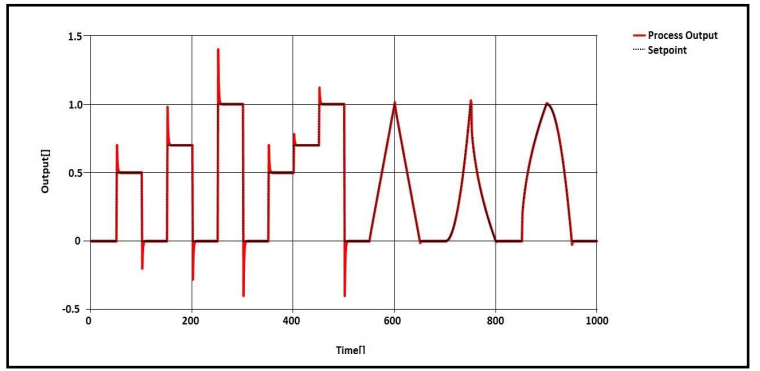

Figure 8. Frist process with tunned PID controller.

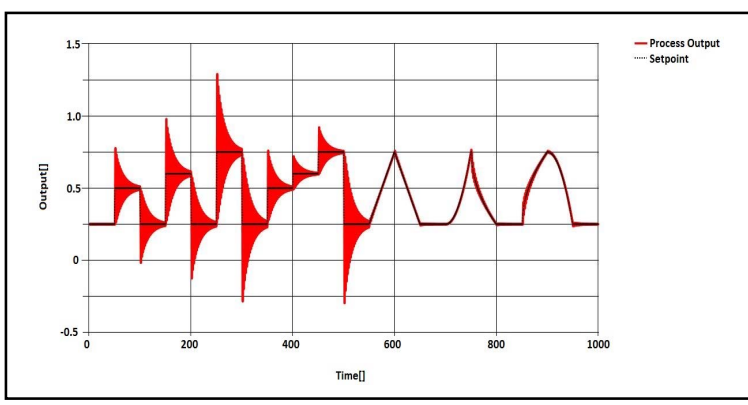

Figure 9. Second process with tunned PID controller.

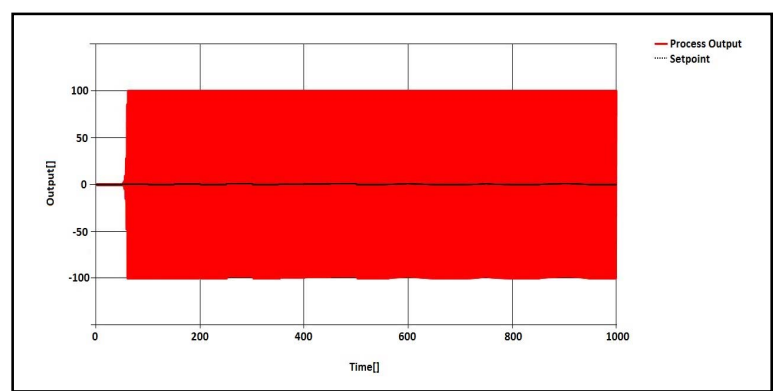

Figure 10. Third process with tunned PID controller.

Accumulative error is estimated by summing up error through simulation. There is shown that tuned PID regulator for one intended process reaches accumulated error about 13 , but if process drifts $10 \%$ (second process) accumulated error is 9 times higher.
(Tab. II) If process drifts more tuned PID controller is insufficient as is shown. (Fig. 10)

TABLE II. ACCUMULATIVE ERROR FOR EACH PROCESS

\begin{tabular}{|c|c|c|c|}
\hline Process & Process_1 & Process_2 & Process_3 \\
\hline Acc. error & 12.98 & 108.45 & $>100000$ \\
\hline
\end{tabular}

\section{ONLINE TUNING METhods}

In this paper is stated state of the art in the field of PID controllers. There are mentioned some tuning methods. Experiment that was created and executed, shows, how error behaves in dynamic process. If some parameter of the process changes, well-tuned PID does not work properly. Accumulated error rises nine-times if process is delayed by 10 percent. If process is changed more PID controller does not work anymore.

Future work is focused on design new adaptive PID controller, which utilize artificial neural network. Tuning PID parameters will be based on variables which influence state and behavior of process.

\section{ACKNOWLEDGMENT}

This work has been supported by Grant No. VEGA 1/0836/16 of the Scientific Grant Agency of the Ministry of Education, Science, Research and Sport of the Slovak Republic.

\section{REFERENCES}

[1] L. Desborough a R. Miller, „Increasing customer value of industrial control performance monitoring - Honeywell's experience," rev. Sixth International Conference on Chemical Process Control, Vol. 98, 2002.

[2] K. J. Astrom a T. Hagglund, PID Controllers: Theory, Design, and Tuning., North Carolina: Research Triangle Park, 1995.

[3] J. C. Shen, „New Tuning Method for PID Controller,“ rev. Proceedings of the IEEE International Conference on Control Applications, Mexico City, 2001.

[4] J. G. Ziegler a N. B. Nichols, ,Optimum setting for automatic controllers," rev. Trans. ASME, vol. 64, 1942.

[5] P. Solatian, S. H. Abbasi a F. Shabaninia, ,Simulation Study of Flow Control Based On PID ANFIS Controller for NonLinear Process Plants,“ rev. American Journal of Intelligent Systems, 2012.

[6] X. Li, F. Yu a Y. Wang, „PSO Algorithm based Online SelfTuning of PID Controller," rev. International Conference on Computational Intelligence and Security, 2007.

[7] M. Clerc a J. Kennedy, „The Particle Swarm: Explosion, Stability, and Convergence in Multi-Dimension Complex Space," rev. IEEE Transactions on Evolutionary Computation, 2002.

[8] P. Mhaskar, N. H. El-Farra a P. D. Christofides, „A Method for PID Controller Tuning Using Nonlinear Control Techniques," rev. Proceeding of the 2004 American Control Conference, Boston, 2004.

[9] D. E. Rivera, M. Morari a S. Skogestad, „Internal model control. 4. PID controller design," rev. Ind. Eng. Chem. Process Des. Dev., 1986. 